\title{
Neuerscheinungen versicherungswissenschaftlicher Bücher - Ausführliche Hinweise
}

\author{
Andrea Uber
}

Angenommen: 12. November 2021 / Online publiziert: 6. Dezember 2021

(C) Springer-Verlag GmbH Deutschland, ein Teil von Springer Nature 2021

Decker, Timo: Die Versicherbarkeit von Geldbußen gegen Verbände. VVW, Karlsruhe, 2021, 216 Seiten. ISBN 978-3-96329-384-9

Die Versicherbarkeit von Geldbußen gegen Verbände, also juristische Personen und Personenvereinigungen, ist im Wesentlichen aus zwei Gründen von hoher praktischer Relevanz. Zum einen sind die Bußgeldrahmen, die Behörden zur Sanktionierung zur Verfügung stehen, in den vergangenen Jahren stetig angehoben worden, beispielsweise im Datenschutz- und im Kartellrecht. Zum anderen steigt vor dem Hintergrund der ARAG-Garmenbeck-Rechtsprechung des BGH die Bereitschaft, Organmitglieder, die für die Geldbußen verantwortlich gemacht werden, in Regress zu nehmen. Beides wirft rechtliche Fragen auf, die zunächst im Arbeits- und im Kartellrecht diskutiert wurden, nun aber unter Geltung der DS-GVO zunehmend auch im Datenschutzrecht eine Rolle spielen, und die in dem vorliegenden Buch behandelt werden. Zunächst wird untersucht, ob sich ein Verband gegen das Risiko von Geldbußen mit Versicherungsschutz eindecken kann. Dabei spielt eine Rolle, ob eine derartige Versicherung überhaupt bürgerlich-rechtlich zulässig wäre. Auch werden verschiedene Marktbedingungen, die in der Cyberrisiko-Versicherung Deckung für Bußgelder unter dem Vorbehalt der rechtlichen Zulässigkeit versprechen und sich gerade im Hinblick auf die hohe Bußgeldandrohung für datenschutzrechtliche Verstöße nach der DS-GVO starker Nachfrage erfreuen, auf ihre Wirksamkeit hin geprüft. Daran an schließt sich die Behandlung der Frage, ob der Verband Organmitglieder für verhängte Geldbußen in Regress nehmen kann, was insbesondere im Wettbewerbsrecht im Zusammenhang mit dem sogenannten Schienenkartell intensiv diskutiert wurde, und ob ein solcher Regress in der D\&O-Versicherung gedeckt werden könnte.

Andrea Uber (四)

Deutscher Verein für Versicherungswissenschaft e. V., Rheinstr. 45-46, 12161 Berlin, Deutschland E-Mail: andrea.uber@dvfvw.de 
Das Buch richtet sich an wissenschaftlich Interessierte gleichermaßen wie an Praktiker aus der Versicherungswirtschaft, der Anwaltschaft und der Justiz, die eine Handhabe zur Bewältigung der vielschichtigen rechtlichen Probleme im Zusammenhang mit der Versicherbarkeit von Geldbußen suchen.

Gudehus, Heinrich: Digitale Geschäftsmodelle in der Versicherungswirtschaft. VVW, Karlsruhe, 2021, 172 Seiten. ISBN 978-3-96329-390-0

Die Digitalisierung erfordert - darin sind sich spätestens nach den Erfahrungen mit der Corana Pandemie alle einig - neue Geschäftsmodelle in Wirtschaft und Gesellschaft. Die Versicherungsbranche als ein datengetriebenes Gewerbe ist davon stärker betroffen als viele andere Wirtschaftsbereiche. Es wäre also zu vermuten, dass gerade sie in der Digitalisierung ihrer Geschäftsmodelle mit gutem Beispiel voranschreitet. In vielen Bereichen interner Prozessverwaltung ist das auch geschehen. Im Hinblick auf die direkt kundenbezogenen Elemente digitaler Geschäftsmodelle ist allerdings noch deutlich Luft nach oben. Das gilt vor allem mit Blick auf die großen digitalen Player, die auf den Feldern Kundenorientierung und Kundenservice in den letzten Jahren ganz neue Maßstäbe gesetzt haben. Vor diesem Hintergrund stellt sich die Frage nach disruptiven Innovationen durch Branchenfremde oder durch Unternehmen aus der eigenen Branche, die aus dem Inland, aber beispielsweise auch aus dem US-amerikanischen oder chinesischen Ausland kommen können. Werden arrivierte Unternehmen mit neuen Geschäftsmodellen, mit neuen Ansätzen das traditionelle Geschäft aufbrechen? Oder schaffen es unabhängige Startups tatsächlich, sich ein Stück aus der Wertschöpfungskette zu sichern?

In dem Buch Konsequent digital werden streng aus einer Kundenperspektive heraus mögliche Alternativen zu den bisherigen Geschäftsmodellen erörtert. Um die Diskussion nicht zu sehr ins Allgemeine abgleiten zu lassen, hat der Autor die Hausratversicherung als eine verbreitete und bekannte Versicherungssparte ausgewählt. Mit ihr zeigt er exemplarisch verschiedene Optionen auf, wie die Digitalisierung im traditionellen Umfeld und darüber hinaus für völlig andere Geschäftsmodelle sorgen könnte - und wohl auch sorgen sollte. Man darf sich nicht täuschen. Corona hat eine Pause für notwendige digitale Transformationsprozesse nicht nur für die Versicherungsbranche gebracht. Danach wird es mit der Digitalisierung von Gesellschaft, Wirtschaft und Verwaltung in einem anderen Tempo weitergehen. Vielleicht sollte die Versicherungswirtschaft diesmal voranschreiten.

Hellwege, Phillip (Hrsg.): Essays on a Comparative History of Fire Insurance, Comparative Studies in the History of Insurance Law/Studien zur vergleichenden Geschichte des Versicherungsrechts (HIL), Band 16, Duncker \& Humblot GmbH, Berlin, 2021, 6 Tabellen, 230 Seiten. ISBN 978-3-428-18372-2

The aim of the present volume is to reassess the history of fire insurance and fire insurance law in selected European countries from comparative perspectives. Its point of departure is the observation that today's state of research is unsatisfactory. Foremost, the history of fire insurance and fire insurance law presents itself differently in the various European historiographies. German authors usually assert that modern fire insurance is rooted in medieval and early modern guild support, and German literature further claims that state-run fire insurance schemes as first 
established towards the end of the seventeenth century were of particular importance for the development of modern fire insurance. By contrast, English literature often treats fire insurance and fire insurance law as being the offspring of marine insurance. Research in many other European countries follows English literature in treating fire insurance as being firmly rooted in marine insurance. The present volume revisits these different narratives.

Hoffmann, Patricia: Telematik-Tarife in der privaten Krankenversicherung. Möglichkeiten der vitaldatenbasierten Tarif-, Prämien- und Vertragsgestaltung. Veröffentlichungen des Instituts für Versicherungswissenschaft der Universität Mannheim, Band 71. VVW, Karlsruhe, 2021, 468 Seiten. ISBN 978-3-96329-393-1

Die Digitalisierung greift in den meisten Industrie- und Geschäftsbereichen, so auch in der Versicherungsbranche, schnell und weit um sich. Neue technische Nutzungsmöglichkeiten verhelfen hier zu innovativen Produktgestaltungen, bei denen zu den bisher üblichen Risikomerkmalen telematisch erhobene und ausgewertete Daten zur Prämiendifferenzierung herangezogen werden. In der privaten Krankenversicherung entstehen mittlerweile erste telematisch geprägte Bonusprogramme, ein erster Anlauf eines vitaldatenbasierten Telematik-Tarifs zeigt sich bei der Kopplung der Erhebung von Fitnessdaten an Prämiennachlässe beispielsweise in einer Berufsunfähigkeitsversicherung. Durch eine Entwicklung telematischer Tarife könnten auch private Krankenversicherer künftig die Möglichkeit erhalten, die Gesundheit und Fitness ihrer Versicherer ständig zu überwachen und in Abhängigkeit gesundheitsbezogener Parameter Boni und Prämiennachlässe gewähren. Möglich erscheinen auch Prognosen bezüglich künftiger Krankheiten und den damit verbundenen finanziellen Bedürfnissen der Versicherten. So könnten die Telematik-Tarife eine gezielte und verbesserte Risikoselektion und eine verbesserte Prozesseffizienz ermöglichen. Dieses Buch untersucht die rechtliche Zulässigkeit und Umsetzbarkeit solcher telematischen Versicherungstarife sowie deren Vereinbarkeit mit der aktuellen Gesetzeslage. Neben der Prüfung versicherungsvertragsrechtlicher und versicherungsaufsichtsrechtlicher Normen werden auch und explizit datenschutzrechtliche Problematiken in den Fokus gerückt. Denn das Erheben und verarbeiten der Vitaldaten unterfällt zumindest teilweise den Normen für Gesundheitsdaten. Die vertragliche Ausgestaltung telematischer Versicherungsverträge sowie die Möglichkeiten einer differenzierteren risikogerechten Prämienkalkulation sind weitere Kernfragen der Arbeit. Das Buch richtet sich sowohl an Studenten der Versicherungswissenschaften als auch an interessierte Praktiker.

Keller, Markus et al.: Einführung in die betriebliche Altersversorgung. 10. Auflage, VVW, Karlsruhe, 2021. ISBN 978-3-96329-355-9

Zwar ist das Betriebsrentenstärkungsgesetz, das letzte ,große“ Reformwerk in der betrieblichen Altersversorgung, bereits 2018 in Kraft getreten, allerdings haben Gesetzgeber, Verwaltung und Gerichte keine Pause eingelegt und seither für weitere wichtige Neuerungen gesorgt. Diese werden alle in der „Jubiläumsauflage“ des bAVStandardwerkes aufgegriffen. Um einige Beispiele zu nennen: Für Versorgungsbezüge gibt es einen neuen Freibetrag in der gesetzlichen Krankenversicherung, für bestimmte Pensionskassen müssen Beiträge zum Pensionssicherungsverein aG ge- 
leistet werden, und die Voraussetzungen für die „,versicherungsförmige Lösung“ wurden vereinfacht. Ebenso wird auf viele Praxisfragen eingegangen. Arbeitgeber interessiert z. B. die korrekte Umsetzung des gesetzlichen Arbeitgeberzuschusses zur Entgeltumwandlung für „Bestandsfälle“, die erst ab 2022 anspruchsberechtigt sind. Oder die Frage, welche Infopflichten man gegenüber den Versorgungsberechtigten beachten muss. Versicherer wiederum interpretieren das Garantieniveau im Rahmen der beitragsorientierten Leistungszusage neu, um im Niedrigzinsumfeld auch weiterhin eine attraktive Verzinsung innerhalb der versicherungsförmigen Durchführungswege sicherstellen zu können. Zu guter Letzt wird die betriebliche Altersversorgung auch ,indirekt“ durch gesetzliche Neuerungen an anderer Stelle tangiert. Die neu geschaffene Grundrente in der gesetzlichen Rentenversicherung kann u. a. durch eine Entgeltumwandlung positiv wie negativ beeinflusst werden. Daher werden auch solche „Randthemen“ im Buch angesprochen.

Die 10. Auflage der „Einführung in die betriebliche Altersversorgung“ dient Experten und Praktikern abseits all dieser aktuellen Themen auch weiterhin als bewährtes Nachschlagewerk. „Anfängern“ wiederum ermöglicht das Buch einen verständlichen und kompakten Einstieg in die komplexe Materie der betrieblichen Altersversorgung. Dazu dienen nicht zuletzt viele Beispiele.

Overbeck, Guntram: Paradoxon der Geldanlage - Kombiniert mit den häufigsten Beratungsfehlern - Wieso? Weshalb? Warum? VVW, Karlsruhe, 2021, 86 Seiten. ISBN 978-3-96329-311-5

Ist das Ziel einer ausreichenden Altersversorgung überhaupt noch erreichbar? Mit welchen Hilfsmitteln kann ein Investor arbeiten, um seine Ziele zu erreichen? Der Kapitalmarkt ist seit 10 Jahren im massiven Umbruch. Die Inflation ist größer als der Zins. Der Bankzins wird teilweise negativ. Viele Kunden wissen nicht mehr, wo investiert werden kann. Berater finden keine Lösungen. In diesem Leitfaden werden genau die Themen aufgegriffen, verknüpft und in einfache Lösungen umgemünzt. Auf anschaulicher Art und Weise werden Sie in die heutige Kapitalwelt eingeführt und geleitet. Lassen Sie sich nicht aufs Glatteis der $0 \%$ Zinsen führen!

Pardey, Frank: Der Haushaltsführungsschaden - Schadenersatz bei Beeinträchtigung oder Ausfall unentgeltlicher Arbeit in Privathaushalten. VVW, Karlsruhe, 2021, 10. Auflage, 322 Seiten. ISBN: 978-3-96329-357-3

Die vorliegenden Entgelttabellen (Stand: April 2021) sind eine unentbehrliche Ergänzung zum Grundwerk „Der Haushaltsführungsschaden“. Im Grundwerk, begründet von Dr. Hermann Schulz-Borck, fortgeführt von Frank Pardey, wird erläutert, wie Schadenersatz bei Beeinträchtigung oder Ausfall unentgeltlicher Arbeit in Privathaushalten zeitlich berechnet wird. Die Entgelttabellen liefern dazu ergänzend die tariflich vorgegebenen Stundenverrechnungssätze, die sich im Tarifvertrag für den öffentlichen Dienst (TVöD) finden und an denen sich auch die Rechtsprechung orientieren kann. Die Anwendung dieser Tabellen wurde vom BGH wiederholt gebilligt. 
Recht, Peter et al.: Lebensversicherungstechnik algebraisch verstehen. Grundstruktur der Kalkulation von Lebensversicherungsverträgen. De Gruyter Studium, De Gruyter Oldenbourg, Berlin, Boston, 2021, 324 Seiten. ISBN:978-3-11074071-4

Das Buch beleuchtet die Kalkulation und die Analyse von Lebensversicherungsverträgen aus technischer Sicht. Es setzt sich zum Ziel, die entsprechenden formalen Zusammenhänge algebraisch zu motivieren und verzichtet darauf, die üblichen Kalkulationsobjekte bzw. die standardisierte Nomenklatur zu verwenden. Ein solcher Blickwinkel führt dann beispielsweise dazu

- Rechnungsgrundlagen als HADAMARD-invertierbare Vektoren aufzufassen,

- Bewertungen mittels des Skalarprodukts darzustellen,

- Lebensversicherungen als Elemente bestimmter Orthogonalräume zu interpretieren oder

- das Deckungskapital als spezielles Element eines affinen Raumes zu identifizieren.

Auf diese Weise wird sich herausstellen, dass sich herkömmliche versicherungstechnische Darstellungen (und die entsprechenden Inhalte) als Spezialisierungen eines viel allgemeineren Zugangs ergeben. Indem hier die algebraischen Zusammenhänge, die die Lebensversicherungstechnik bestimmen, in den Vordergrund gerückt werden, ergibt sich ein (zusätzliches) Verständnis für die aktuariellen Eigenschaften, die mit einem Lebensversicherungsvertrag verbunden sind.

Salaris, Jeffrey: Eine Kausalanalyse von Erwartungen und ihren Einflussfaktoren im Kontext der Qualitätsbeurteilung von Altersvorsorgeberatungen. Studien zum Konsumentenverhalten, Band 89, Verlag Dr. Kovač, Hamburg, 2021, 418 Seiten. ISBN 978-3-339-12482-1 (Print) und ISBN 978-3-339-12483-8 (eBook)

Die Rentenstrukturreform 2001 und der damit verbundenen Verabschiedung des Altersvermögensgesetzes (AVmG) und des Altersvermögensergänzungsgesetzes (AVmEG) war eine Reaktion des Gesetzgebers auf die demographische Entwicklung. So wird die private Altersvorsorge spezifisch durch steuerliche Maßnahmen gefördert, während das Leistungsniveau der gesetzlichen Rentenversicherung reduziert wird. Mit dem prognostizierten langfristigen Leistungsniveau der gesetzlichen Rentenversicherung ist bereits jetzt erkennbar, dass die Leistungen der GRV nur noch eine armutsvermeidende Mindestversicherung darstellen werden. Um die Stetigkeit und Sicherheit des Einkommensbezuges beim Übergang von Erwerbs- in die Nacherwerbsphase zu gewährleisten, müssen die Leistungen aus der gesetzlichen Rentenversicherung zwingend durch Leistungen aus betrieblicher und/oder privater Vorsorge ergänzt werden. Dadurch wurde von den Haushalten verstärkt eigenverantwortliches Handeln zur Sicherung des Alterseinkommens gefordert. Finanzentscheidungen, wie beispielsweise Altersvorsorgeentscheidungen, stellen ein Individuum unter anderem aufgrund mangelnder finanzieller Kenntnisse, der Langfristigkeit des Vertrages, der Vielzahl an Anbietern und der wahrgenommenen Komplexität der Produkte oftmals vor eine schwierige Entscheidungssituation. Daraus leitet sich ein enormer Bedarf an Altersvorsorgeberatung ab. Auch im versicherungswissenschaftlichen Kontext wird die Erwartung meist als gegebener Referenzpunkt hingenommen und eine wissenschaftliche Auseinandersetzung 
hinsichtlich ihrer Bedeutung für die Qualitätswahrnehmung erfolgt nicht. Aus diesem Grund werden in der vorliegenden Dissertationsschrift die Einflussfaktoren der Erwartungsbildung hinsichtlich der Qualitätsbeurteilung von Altersvorsorgeberatungen tiefergehend betrachtet. So werden die Wirkungszusammenhänge der Einflussfaktoren Beratungserfahrung, finanzielle Allgemeinbildung, kognitives Involvement, affektives Involvement und Einstellung im Hinblick auf die Erwartungen hinsichtlich der Qualität einer Altersvorsorgeberatung analysiert. Hierfür werden die Konzepte der mehrdimensionalen Toleranzzone und des Erwartungs-Diskonfirmations-Paradigmas zusammengeführt und mittels Kausalanalysen untersucht.

Allerdings war die Qualität der Altersvorsorgeberatung in den vergangenen Jahren ebenfalls mehrfach im Mittelpunkt der gesellschaftlichen und politischen Diskussion. In dieser Diskussion fehlt jedoch ein gemeinsames Verständnis, wie Beratungsqualität $\mathrm{zu}$ verstehen oder $\mathrm{zu}$ messen ist. In der wissenschaftlichen Literatur dient das sogenannte Erwartungs-Diskonfirmations-Paradigma als Basis für die Qualitätswahrnehmung. In einem Vergleichsprozess werden die Kundenerwartungen an eine Leistung (Soll-Standard) mit der tatsächlich wahrgenommenen Leistung (Ist-Leistung) verglichen. Dieser Ansatz wird auch im Qualitätsmanagement angewendet, wobei sich in der Praxis ein klarer Schwerpunkt auf die Optimierung der wahrgenommenen Leistung herausgebildet hat. Durch die Fokussierung auf die Leistungsseite werden allerdings die Potenziale zur Erreichung einer hohen Qualitätswahrnehmung auch auf der Erwartungsseite nicht ausgeschöpft.

Scharff, Hendrik: Das (neue) Provisionsabgabeverbot für Versicherungsvermittler Konzept, Funktion und Grenzen. VVW, Karlsruhe, 2021, 290 Seiten. ISBN 978-396329-381-8

Mit dem Gesetz zur Umsetzung der Versicherungsvertriebsrichtlinie (Richtlinie (EU) 2016/97) hat der deutsche Gesetzgeber ein völlig neu gestaltetes Sondervergütungs- und Provisionsabgabeverbot eingeführt. Damit wurde eine neue Rechtsgrundlage für den Versicherungsvertrieb geschaffen, die allerdings weder richtlinienbasiert ist, noch eine Parallele in einem anderen Mitgliedstaat der europäischen Union aufweist. Durch das Verbot sollen Verbraucherinnen und Verbraucher vor Fehlanreizen durch Versicherungsunternehmen und Versicherungsvermittler geschützt werden. Ein derartiges Verbot ist allerdings äußerst ungewöhnlich, weil die Abgabe eines Teils der eigenen Vergütung, also der Verzicht auf Ertragsanteile, im Wirtschaftsleben abseits der Versicherungsbranche keine Frage des Rechts, sondern allein eine Frage der Geschäftspolitik ist. Zugleich hemmt das Verbot innovative, neue Vermittlungskonzepte, wie sie z. B. durch Online-Versicherungsvermittler entwickelt werden.

In seiner Dissertation untersucht der Verfasser u.a., ob der Gesetzgeber mit dem Sondervergütungs- und Provisionsabgabeverbot das von ihm verfolgte Ziel des Verbraucherschutzes erreicht und ob das Verbot europarechtlich und verfassungsrechtlich gerechtfertigt ist. Hierzu analysiert er umfassend das Konzept des Gesetzes vor dem Hintergrund der tatsächlichen und rechtlichen Gegebenheiten auf dem Markt für Versicherungsvermittlung und unterzieht es einem Vergleich mit strukturverwandten Vermittlermärkten. Anhand der so gewonnenen Erkenntnisse stellt der Verfasser die gesetzgeberischen Grundannahmen über den Zweck und die Wirkungsweise 
des Provisionsabgabeverbots auf den Prüfstand und analysiert dessen Funktion und rechtliche Grenzen. Das Werk richtet sich damit zum einen an Rechtswissenschaft und Rechtspraxis. Zum anderen bietet die Arbeit mit ihrem umfassenden Beitrag zum Konzept des Provisionsabgabeverbots eine Handreichung für den Versicherungsvertrieb.

Schwepcke, Andreas et al.: Praxishandbuch Rückversicherung. 2. Auflage, VVW, Karlsruhe, 2021, 580 Seiten. ISBN 978-3-96329-337-5

Das Praxishandbuch Rückversicherung - ein völlig neues Handbuch! Damit gibt es endlich eine profunde Einführung in einen bedeutenden Themenkreis - und ein Nachschlagewerk für alle speziellen Problemstellungen rund um die Rückversicherung. Dieses brandneue Handbuch kann abschnittsweise oder sogar komplett als methodisch durchdachte Einführung in die Rückversicherung gelesen werden. Denn hier wird besonderer Wert auf die Vermittlung von Basiswissen und Methodik der Rückversicherung gelegt. Das Praxishandbuch ist in elf Abschnitte untergliedert: Dabei geht es um geschichtliche Ursprünge der Rückversicherung, um ihre wirtschaftliche Bedeutung, um maßgeblichen Rechtsgrundlagen und um die Funktionsweisen der verschiedenen Rückversicherungsformen und -arten. Berücksichtigt werden dabei ganz besonders der sachliche, zeitliche und geografische Deckungsumfang sowie alle einschränkenden Klauseln. Ein solches Praxishandbuch hat nicht nur den Profis in den Versicherungsunternehmen gefehlt: Hier werden die Unterschiede zwischen traditionellen und alternativen Rückversicherungsformen aufgezeigt, gegenübergestellt werden aber auch Rückversicherungsbedarf und -politik des Erstversicherers mit der Zeichnungskapazität und -politik des Rückversicherers unter Beachtung einzelner Sparten und deren Besonderheiten. Weitere Themenschwerpunkte sind u. a.: Entscheidungsgrundlagen für die Gestaltung von Rückversicherungsprogrammen Zwecke und Quellen von Rückversicherungsstatistiken - Methoden der Preisermittlung und Preiserhebung - Rückversicherungs-Underwriting - Schaden- und RunOff-Management in der Rückversicherung. Abschließend wird auf die Grundbegriffe der Rechnungslegung und die Wirkung von Rückversicherung auf die Bilanzen der Vertragsparteien und deren Solvabilitätsvorgaben unter Solvency II eingegangen, wobei auch Aspekte der Risikobewältigung beschrieben werden. Damit richtet sich das vorliegende Handbuch der Rückversicherung also wirklich an alle, die sich mit Rückversicherung beschäftigen oder es tun wollen. Anfänger können sich in eine komplexe Materie einarbeiten, während Profis die Grundlage für den vertieften Einstieg im gebotenen Detail haben. Auch für das Studium zum Versicherungsfachwirt unentbehrlich! Mit dem umfangreichen Stichwortverzeichnis auch das ideale Nachschlagewerk!

Straubhaar, Thomas: Grundeinkommen - Jetzt - Nur so ist die Marktwirtschaft zu retten. NZZ Libro, Schwabe Verlagsgruppe AG, Basel, 2021, 284 Seiten. ISBN 9783-907291-52-8 (Print), ISBN 978-3-907291-53-5 (E-Book)

Zeitenwenden erfordern einen Geisteswandel. Es braucht keine Revolution. Kapitalismus und Marktwirtschaft müssen nicht zertrümmert werden. Wer das Bewährte erhalten will, muss aber bereit sein, einiges zu verändern. Zentrale Aspekte unseres Lebens müssen wir überdenken und den neuen Gegebenheiten anpassen. 
Das bedingungslose Grundeinkommen ist mehr als eine utopische Reaktion auf die dystopischen Erfahrungen der Corona-Pandemie. Es liefert ein neu ausbalanciertes Zusammenspiel von individueller Entscheidungsfreiheit und der Notwendigkeit, sich als Solidargemeinschaft gegen systemische Grossrisiken zu versichern. Perfekt ist das nicht, aber besser als jede Alternative, wenn es um eine Anpassung des Sozialstaats an disruptive Zeiten geht.

Wer will, kann. Die Pandemie hat gezeigt, dass nahezu alles geht, wenn Not scheinbar keine andere Wahl lässt. Ein bedingungsloses Grundeinkommen ist die europäische Antwort, um mit einer Kultur von Mass und Mitte, Kompromiss und Ausgleich, Mitsprache und Mitbestimmung gegen amerikanische Dominanz und chinesische Machtansprüche erfolgreich bestehen zu können. Prof. Thomas Straubhaar, Professor für Volkswirtschaftslehre mit Schwerpunkt internationale Wirtschaftsbeziehungen an der Universität Hamburg, zeigt, wie eine neue Arbeitsteilung zwischen Markt und Staat aussehen kann. Er weist der Marktwirtschaft des 21. Jahrhunderts den Weg.

Trakic, Adnan (Hrsg.): Covid-19 and Business Law. Legal Implications of a Global Pandemic. De Gruyter Studies in Global Asia, Band 3. De Gruyter, Berlin, Boston, 2021, 187 Seiten. ISBN 978-3-11072369-4

The COVID-19 pandemic has had extraordinary effects on human lives and economies around the world. Many countries have introduced various measures to stop the spread of the virus and preserve human lives and livelihoods. Some commentators have considered these measures extreme, such as the restrictions imposed on people's movement and lockdown of countries' borders. While these measures have undoubtedly saved lives and curbed the spread of the deadly virus, they have also produced some unintended legal implications for individuals and businesses, particularly in the areas of contractual obligations, employment relationships, tourism and hospitality, company law, competition law, human rights and the rule of law, protection of vulnerable groups like migrant workers, and access to judicial and legal services. COVID-19 and Business Law: Legal Implications of a Global Pandemic identifies and discusses specific legal challenges caused by the COVID-19 pandemic in these areas and suggests possible ways in which they could be remedied.

- Offers insights into the legal impacts of the COVID-19 pandemic on individuals and businesses

- Provides comprehensive legal solutions and suggests law reforms where considered necessary

- Includes laws and examples from several jurisdictions

Wein, Fabian: Die Geschichte der privaten Krankenversicherung 1945-1994. Studien zur vergleichenden Geschichte des Versicherungsrechts, Band 17. Duncker \& Humblot, Berlin, 234 Seiten. ISBN 978-3-428-18388-3 (Print), ISBN 978-3-42858388-1 (E-Book)

Trotz der seit 1945 fortdauernder Debatte über die Rolle der PKV ist ihre Entwicklung bis zur Deregulierung im Jahr 1994 nur punktuell aufgearbeitet. Die gesellschaftliche Rolle der PKV und ihr Verhältnis zur GKV waren stets umstritten. Trotz 
der hohen Regulierungsdichte konnte sich die PKV auch durch eine Vielzahl an eigenständigen Innovationen behaupten. Insbesondere die Entwicklung der Krankheitskostenvolltarife und die Ausgestaltung der Zusatzversicherungstarife können nachvollzogen werden. Dem nach dem Ende des Wirtschaftswunders rapide zunehmendem Kostendruck konnte die PKV besser als die GKV widerstehen. In der Folge übernahm die PKV verstärkt sozialpolitische Verantwortung und es kam zu einer Annäherung der beiden Systeme. Im Rahmen der fortlaufenden Reformen übertrug der Gesetzgeber Elemente der PKV in die Gesetzliche Krankenversicherung und die PKV entwickelte ihrerseits sozialschützende Elemente jenseits ihrer klassischen, privatrechtlichen Wurzeln.

Zampano, Laura: Die Ursprünge der Versicherungsaufsicht in Deutschland und Italien aus historisch-vergleichender Perspektive. Studien zur vergleichenden Geschichte des Versicherungsrechts, Band 15. Duncker \& Humblot, Berlin, 2021, 210 Seiten. ISBN 978-3-428-18301-2 (Print), ISBN 978-3-428-58301-0 (E-Book)

Die Geschichte des Versicherungsaufsichtsrechts ist bisher nur punktuell erforscht worden. Die deutsche Forschung hat sich intensiv mit dem Thema auseinandergesetzt, geht aber selten auf die Zeit vor 1781 zurück. Für andere europäische Rechte fehlen historische Darstellungen der Versicherungsaufsicht dagegen fast vollständig, so auch für das italienische Recht. In dieser Arbeit wird die historische Entwicklung der Versicherungsaufsicht in Deutschland und Italien untersucht und gegenübergestellt. Für beide Länder werden, neben versicherungsaufsichtsrechtlichen Vorschriften im modernen Sinne, auch solche Vorschriften, die vor der Einführung einer echten Versicherungsaufsicht verabschiedet wurden, erforscht, um die Ursprünge, die zur versicherungsaufsichtsrechtlichen Gesetzgebung im eigentlichen Sinne geführt haben, zu rekonstruieren. 